Check for updates

Cite this: Phys. Chem. Chem. Phys., 2018, 20, 13952

Received 12th February 2018 Accepted 27th April 2018

DOI: $10.1039 / c 8 c p 01008 k$

rsc.li/pccp

\title{
Counterion binding alters surfactant self-assembly in deep eutectic solvents $\dagger$
}

\author{
A. Sanchez-Fernandez, (iD *ab O. S. Hammond, (iD c K. J. Edler, (iD $\star^{a}{ }^{\mathrm{a}}$ T. Arnold, (iD abd \\ J. Doutch, ${ }^{e}$ R. M. Dalgliesh, (D) ${ }^{e}$ P. Li, ${ }^{e}$ K. Ma ${ }^{e}$ and A. J. Jackson (iD bf
}

\begin{abstract}
Micellisation of surfactants in deep eutectic solvents has been recently demonstrated to provide a controllable way to modify micelle morphology. Ion-pair interactions between the solvent and the surfactant headgroup were identified as affecting the micellisation by modifying the charge density of the micelle. Here we explore the micellisation of dodecylsulfate surfactants with different counterions $\left(\mathrm{Li}^{+}, \mathrm{Cs}^{+}, \mathrm{Mg}^{2+}, \mathrm{Bmim}^{+}, \mathrm{Emim}^{+}, \mathrm{Cholinium}^{+}\right)$dissolved in two deep eutectic solvents: choline chloride:urea and choline chloride:glycerol. Surface tension results show a solvent and counterion dependence of the CMC of the surfactants. Small-angle neutron scattering was subsequently used to investigate the morphology of the micelles formed. The results show that the elongation of the micelles is strongly dependent on the solvent, showing more elongated aggregates in choline chloride:urea than in choline chloride:glycerol. The morphology of micelles in DES was also found to depend on the counterion, where the affinity of binding showed similarities to that in water.
\end{abstract}

\section{Introduction}

Deep eutectic solvents (DES) are sustainable ionic solvents, normally formed through the complexation of a halide salt (most frequently choline chloride) with an organic hydrogen bond donor (such as urea or glycerol). ${ }^{1}$ The resulting mixture is in a high-entropy state which remains liquid at attractively low temperatures, where the formation and stability of the solvent relies on an extensive ensemble of hydrogen bond interactions between the various components. ${ }^{2-5}$ Furthermore, the characteristics of the resulting solvent can be tailored through different combinations of precursors, allowing the solvent to be designed for better performance in a particular application. ${ }^{1,6}$ Although many recent developments within the field of DES have been focused on finding new applications for these solvents, many questions about DES remain unclear due to limited

\footnotetext{
${ }^{a}$ Department of Chemistry, University of Bath, Claverton Down, Bath, BA2 7AY, UK. E-mail: a.sanchez.fernandez@bath.ac.uk,k.edler@bath.ac.uk

${ }^{b}$ European Spallation Source, Lund, SE-221 00, Sweden

${ }^{c}$ Centre for Sustainable Chemical Technologies, University of Bath, Claverton Down, Bath, BA2 7AY, UK

${ }^{d}$ Diamond Light Source, Harwell Campus, Didcot, OX11 ODE, UK

${ }^{e}$ ISIS Neutron and Muon Source, Science and Technology Facilities Council, Rutherford Appleton Laboratory, Didcot, OX11 OQX, UK

${ }^{f}$ Department of Physical Chemistry, Lund University, Lund, SE-221 00, Sweden $\dagger$ Electronic supplementary information (ESI) available: Surfactant synthesis and characterization, surface tension data, SANS model test, Guinier analysis and results from isotopic mixtures not shown in the main text. Data supporting this article has been made freely available via the University of Bath Research Data Archive system at DOI: 10.15125/BATH-00500. See DOI: 10.1039/c8cp01008k
}

investigations into their fundamental behaviour. Therefore, unexpected applications are likely to emerge from future investigations into the fundamental physicochemical properties of DES systems.

Surfactant molecules consist of both lyophilic and lyophobic moieties. This amphiphilic structure drives a particular behaviour when in solution, that leads to the self-assembly of aggregates above a certain concentration, known as the critical micelle concentration (CMC). The aggregates, commonly deemed micelles, are formed through the aggregation of surfactant in order to reduce the free energy of the system. The energetically unfavourable lyophobic interaction between tail and solvent is overcome by forming aggregates where the tails are relatively non-solvated by the surrounding medium, because the solvated headgroups form a shell protecting the tails. ${ }^{7-9}$ A fine balance between the different parts of the system controls the characteristics of those aggregates, where repulsion - either steric or electrostatic - limits the size that the micelle attains. In particular, the morphology of ionic surfactant micelles is controlled by the repulsion between ionised headgroups. The presence of counterions near the headgroup region controls the charge density and, depending on the characteristics of the counterion, the effective charge and hence structure of the micelle.,10

Deep eutectic solvents (DES) have recently been shown to support surfactant self-assembly, ${ }^{11,12}$ solvation of polymers, ${ }^{13,14}$ protein folding, ${ }^{15}$ and formation of phospholipid vesicles; ${ }^{16,17}$ an unusual set of characteristics for a non-aqueous solvent. The aggregation of surfactant was found to be particularly interesting due to the open possibilities towards developing controllable self-assembly. Two main routes to promote self-driven aggregation 
in DES have been identified: non-interacting and interacting self-assembly. The non-interacting route shows similarities with traditional self-assembly in pure molecular solvents, where the interaction between the headgroups in a polar environment controls the morphology of the micelle, corresponding to a certain amount of adsorbed counterions. ${ }^{11}$ Interacting self-assembly is characterised by the formation of different morphologies to those seen in pure solvents. Interactions between components of the solvent and sodium dodecylsulfate (SDS), a prototypical anionic surfactant, were hypothesised to play a key role in the transition of the aggregates from globular micelles in a neutral environment ${ }^{18}$ to elongated micelles in choline chloride:urea. ${ }^{19}$ These systems are of particular interest due to the tuneability of the micelle morphology, opening novel approaches for applications in surfactant templating of nanostructured materials, drug delivery and stimuli-responsive fluids. However, the following questions remain unclear regarding the nature of the solvation of the headgroup region in interactive self-assembly and the effects on micelle morphology: are the choline ions the only species interacting with the headgroups, or is the native surfactant counterion present in the Stern layer; and is this a general case in any choline-based DES, or is there a composition-dependence?

The aim of this study is to evaluate the role of different counterions on the micellisation of dodecylsulfate surfactants and increase our understanding of micellisation in DES. Here we investigate the effect of different counterions on the CMC of those surfactants and the micelle morphology in choline chloride: urea and choline chloride:glycerol. Surface tension and smallangle neutron scattering (SANS) have been used to investigate the behaviour of various counterion exchanged surfactants. Two main categories were investigated, including inorganic counterions: lithium dodecylsulfate (LiDS), cesium dodecylsulfate (CsDS) and magnesium didodecylsulfate $\left(\mathrm{Mg}_{1 / 2} \mathrm{DS}\right)$; and organic counterions: 1-ethyl-3-methylimidazolium dodecylsulfate (EmimDS), 1-butyl3-methylimidazolium dodecylsulfate (BmimDS) and choline dodecylsulfate (ChDS). The structure of the investigated surfactants is presented in Fig. 1. The results from these systems were interpreted in terms of counterion condensation on the micelle interface and compared with analogous results in aqueous solution and ionic liquids.

\section{Experimental}

\section{Materials}

DES were prepared the standard procedure explained elsewhere. ${ }^{20,21}$ Hydrogenated choline chloride ( $\mathrm{ChCl},>99 \%$, Acros Organics), glycerol (Glyc, 99\%, Sigma-Aldrich) and urea (>99\%, SigmaAldrich) were purchased and used without further purification. d9-Choline chloride (99\% purity, 99.6\% D), d8-glycerol (99\% purity, 99\% D) and d4-urea (99\% purity, 98\% D) were supplied by CK isotopes and used without further purification. Solvents were freeze-dried, sealed and stored under a dry atmosphere to minimise water absorption. Isotopically substituted DES were prepared following the same procedure explained above. Water content was determined, using a Mettler-Toledo DL32 Karl

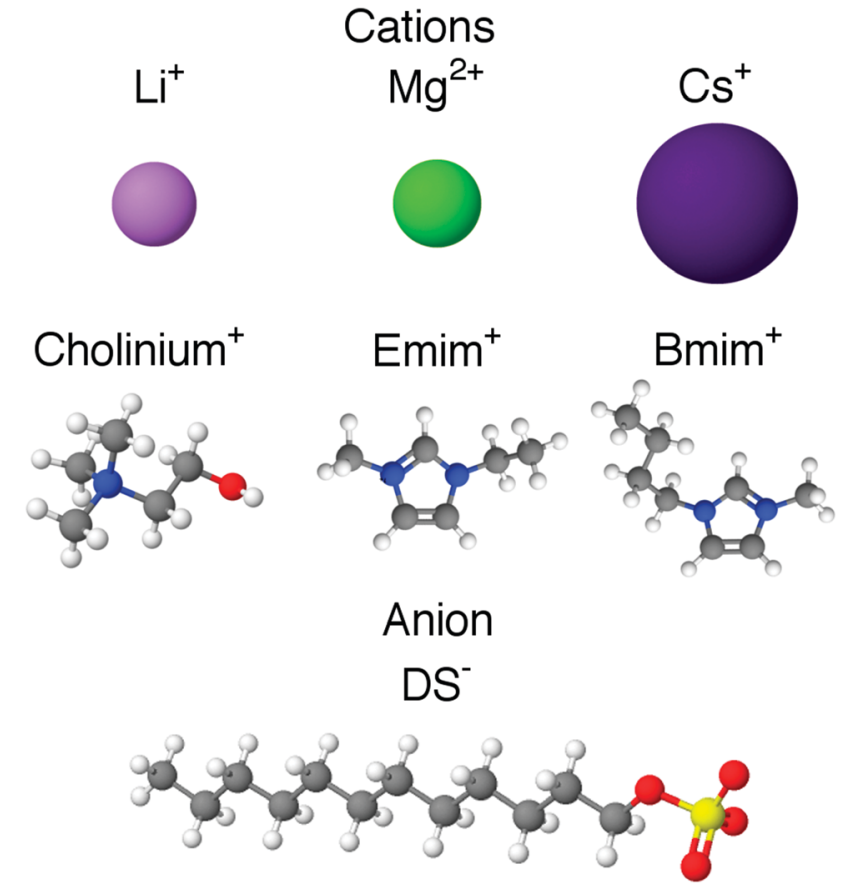

Fig. 1 Structure of the inorganic and organic cations, and the common dodecylsulfate anion, that were the subject of this investigation.

Fischer titrator, to have an average water content of 0.32 and $0.22 \mathrm{wt} \%$, for choline chloride:glycerol and choline chloride:urea respectively during the experimental procedure presented here.

Protonated sodium dodecylsulfate (SDS, 99\%, Sigma) and lithium dodecylsulfate (LiDS, 99\%, Alfa Aesar) were purchased and used without further purification. Cesium dodecylsulfate (CsDS), magnesium didodecylsulfate $\left(\mathrm{Mg}_{1 / 2} \mathrm{DS}\right)$, choline dodecylsulfate (ChDS), 1-ethyl-3-methylimidazolium dodecylsulfate (EmimDS) and 1-butyl-3-methylimidazolium dodecylsulfate (BmimDS) were prepared by counterion exchanging SDS, following the procedures detailed in the ESI. $\dagger^{22-24}$ Deuterated versions of sodium dodecylsulfate (d-SDS), lithium dodecylsulfate (d-LiDS) and magnesium didodecylsulfate $\left(\mathrm{d}-\mathrm{Mg}_{1 / 2} \mathrm{DS}\right)$ were supplied by ISIS Deuteration Facility. Deuterated caesium dodecylsulfate was prepared from d-SDS following the same procedure as described above. The purity of the surfactants was assessed by ${ }^{1} \mathrm{H}-\mathrm{NMR}$ and sodium atomic absorption spectroscopy. Details about the characterisation can be found in the ESI. $\dagger$

\section{Methods}

Surface tension. The CMCs were determined by means of surface tension. Surface tension measurements of different concentrations of surfactant below and above the CMC were performed using the pendant-drop method using a Kruss DSA100. Samples were equilibrated at $50{ }^{\circ} \mathrm{C}$ prior to measurements. Several drops of each sample were suspended using a $1.82 \mathrm{~mm}$ needle and images recorded after drop-shape equilibration. The contour of the drop was fitted using the Young-Laplace equation to obtain the surface tension of the sample. This procedure was repeated at least 5 times for each sample and the end results were averaged from the individual measurements. Although temperature 
could not be controlled during the experimental procedure, the method used here was found to be self-consistent.

Samples for surface tension measurements of these surfactants in DES were prepared by dilution of stock solutions to reduce variability. A high concentration stock solution was prepared by direct mixing of each surfactant in the corresponding solvent. The solutions were equilibrated for at least $24 \mathrm{~h}$ at $50{ }^{\circ} \mathrm{C}$ and subsequently diluted down using the pure solvent to obtain a range of various concentrations below and above the CMC. These samples were sealed and stored under a dry atmosphere to prevent the adsorption of water from the environment.

Small-angle neutron scattering measurements. SANS experiments were performed on Sans $2 \mathrm{~d}^{25}$ and Larmor at ISIS Pulsed Neutron Source, UK. Sans2d is a time-of-flight instrument with two movable detectors. The rear detector was placed at $4 \mathrm{~m}$ distance from the sample position, giving a total momentum transfer $(q)$ range of $0.004-0.9 \AA^{-1}$. Larmor is a versatile time-of-flight neutron instrument. When operated in SANS mode, a fixed position detector is placed at $4 \mathrm{~m}$, providing a $q$-range of $0.003-0.8 \AA^{-1}$.

Different concentrations above the CMC of each surfactant were prepared for SANS experiments by direct mixing of the DES with the surfactants. These samples were sealed and equilibrated in an oven at $40{ }^{\circ} \mathrm{C}$ overnight. After equilibration, samples were sealed and stored until the experiments were performed. For all the scattering patterns from surfactants with inorganic counterions $\left(\mathrm{Li}^{+}, \mathrm{Cs}^{+}\right.$and $\left.\mathrm{Mg}^{2+}\right)$ in both solvents, the structural parameters of the model were co-refined for two contrasts: d-MDS + h-ChCl:h-Glyc or h-ChCl:h-urea and h-MDS + d-ChCl:d-Glyc or d-ChCl:d-urea. The volume fraction of scatterers was individually fitted to account for small variations in the volume fraction of micelles between contrasts. Data from organic counterions $\left(\mathrm{Emim}^{+}, \mathrm{Bmim}^{+}, \mathrm{Ch}^{+}\right)$were collected in one contrast and individually fitted: h-MDS + d-ChCl:d-Glyc or d-ChCl:d-urea.

For both experiments, samples were loaded in $1 \mathrm{~mm}$ path length, $1 \mathrm{~cm}$ width, quartz Hellma cells and these were placed in a temperature-controlled sample changer at $50{ }^{\circ} \mathrm{C}$ during data collection. Data were reduced using the standard routines of Mantid. ${ }^{26}$ Data were normalised to sample transmission and corrected for detector efficiencies and the scattering from an empty cell. ${ }^{27}$ The scattering of the pure solvents was subtracted accounting for the incoherent contribution to each sample. ${ }^{28}$ The output data was absolute scattered intensity, $I(q)$ in $\mathrm{cm}^{-1}$, versus the momentum transfer, $q$ in $\AA^{-1}$.

Small-angle neutron scattering analysis. As a first approach to data fitting, the Guinier approximation was used to determine the radius of gyration $\left(R_{\mathrm{g}}\right)$ of the scatterers. This approach was used with samples in the diluted regime to avoid the interference of intermicellar scattering. The Guinier approximation allows the calculation of the size of the scatterer by analysing the low- $q$ expansion of the data in a $\ln (I) v s . q^{2}$ plot. In this regime, the $R_{\mathrm{g}}$ of the scattering object can be calculated from the slope of the graph

$$
\ln (I(q))=\ln (I(0))-\frac{R_{\mathrm{g}}{ }^{2}}{3} q^{2}
$$

Further structural information from small-angle neutron scattering data was derived through model-based fitting. The scattered intensity in the small-angle region of isotropic, centrosymmetric particles can be described as follows:

$$
I(q)=N V^{2}(\Delta \mathrm{SLD})^{2} P(q) S(q)+B
$$

where $N$ is the number of particles and $V$ their volume. $\triangle \mathrm{SLD}$ corresponds to the scattering length density contrast between particles and solvent. $P(q)$ is a $q$-dependent model that accounts for the form factor of the particles, whilst $S(q)$ is the structure factor and accounts for the interaction between particles.

To identify the best model to account for the form factor contribution, different geometric models (monodisperse and polydisperse spheres, monodisperse ellipsoids and monodisperse cylinders) were tested (see ESI $\dagger$ ). ${ }^{29}$ An ellipsoid model with prolate distribution of mass was found to be the best model to account for the morphology of individual particles. This model describes a uniform ellipsoid shape and variations in the aspect ratio ( $\mathrm{AR}=r_{\mathrm{po}} / r_{\mathrm{eq}}$, where $r_{\mathrm{po}}$ is the polar radius of the scatterer and $r_{\text {eq }}$ is the equatorial radius) are intrinsically correlated with the shape of the micelle as follows: spherical micelles when $\mathrm{AR}=1\left(r_{\mathrm{po}}=r_{\mathrm{eq}}\right)$, globular micelles when $\mathrm{AR}>1$ $\left(r_{\mathrm{po}}>r_{\mathrm{eq}}\right)$ and elongated aggregates when AR $\gg 1\left(r_{\mathrm{po}} \gg r_{\mathrm{eq}}\right)$. This approach allows direct comparison between different systems and immediate evaluation of their characteristics. This model contains the following parameters: equatorial radius, aspect ratio and volume fraction of scatterers $\left(\phi_{P(q)}\right)$. No polydispersity factor was included in the model as micelles in thermodynamic equilibrium should show a low polydispersity ${ }^{30}$ and it was not required to obtain a good fit to the data.

SANS data from the various surfactant solutions at low concentration were fitted to this model between 0.006 and $0.5 \AA^{-1}$ of momentum transfer, using the Levenberg-Marquardt algorithm incorporated into SasView. ${ }^{31}$ The resolution of the instrument was accounted for by smearing of the mathematical model using a Gaussian function at a constant $8 \% \mathrm{~d} q / q$. The scattering length density (SLD) of each component of the system was calculated by accounting for the scattering length of the atomic group $\left(\sum b_{\mathrm{i}}\right)$ and the occupied volume by this group $\left(V_{\mathrm{m}}\right)$. Assuming that the contribution of the headgroup to the scattering will be rather small due to solvation, the SLD of the surfactant was calculated as the lyophobic tail (without considering the sulfate headgroup) and therefore the same value was used to fit all the investigated surfactants. ${ }^{12,32}$ Because of this consideration, the SLDs of solvent and particle are fixed during fitting. Molecular volumes and scattering length densities of the DES were used as previously reported. ${ }^{11,19}$ Scattering lengths, molecular volumes and SLDs are presented in Table 1.

A modified Percus-Yevick hard-sphere approximation was used to account for the structure factor interaction between micelles in concentrated systems. ${ }^{11,19,28}$ Previous investigations on intermicellar interactions in DES have presented the remarkable difficulties when evaluating the structure factor. Although various models were previously tested (rescaled mean square approximation, mean square approximation and hard sphere structure factor), ${ }^{33-35}$ none of these were found to satisfactorily account for the intermicellar contribution to the scattering. Therefore a methodology to fit the structure factor 
Table 1 Volumes, scattering lengths and scattering length densities of constituents of the systems

\begin{tabular}{|c|c|c|c|}
\hline Component & $V_{\mathrm{m}} / \AA^{3}$ & $\sum b_{\mathrm{i}} / \mathrm{fm}$ & $\mathrm{SLD} / 10^{-6} \AA^{-2}$ \\
\hline $1: 2 \mathrm{~h}$-ChCl:h-urea & 343 & 44.5 & 1.2 \\
\hline $1: 2 \mathrm{~d}-\mathrm{ChCl}: \mathrm{d}-$ urea & 343 & 221 & 6.2 \\
\hline 1:2 h-ChCl: h-Glyc & 453 & 20.4 & 0.45 \\
\hline 1:2 d-ChCl:d-Glyc & 453 & 281 & 6.3 \\
\hline h-DS ${ }^{-}$ & 409 & 16.0 & -0.39 \\
\hline $\mathrm{d}-\mathrm{DS}^{-}$ & 409 & 276 & 7.0 \\
\hline
\end{tabular}

was developed in order to deconvolute the form factor and correctly evaluate the morphology of the aggregates. ${ }^{11,19,28}$ This structure factor is described by two parameters that are not constrained to the values of the form factor. An effective radius $\left(R_{\text {eff }}=\left(r_{\mathrm{po}} r_{\mathrm{eq}}{ }^{2}\right)^{1 / 3}\right)$ accounts for the radius of interaction as a rigid sphere of radius $R_{\text {eff }}{ }^{36,37}$ The second parameter, a volume fraction associated with the $S(q)\left(\phi_{S(q)}\right)$, accounts for the volume fraction of the repulsive interaction. This approach allows the evaluation of the structure factor contribution to the scattering and therefore we can consistently evaluate the form factor of the micelles. However, this descriptive approach does not use an analytical model and the micelle-micelle interaction is not yet well understood. Hence direct physical interpretation of the interaction between micelles cannot be obtained.

\section{Results}

\section{Critical micelle concentrations}

Surface tension is a useful technique to determine the critical micelle concentration (CMC) of surface-active molecules. The CMCs of the surfactants measured in DES here are presented in Table 2 alongside literature values for water, where available. The as-measured surface tension plots from which the CMC of these systems was derived are included in the ESI. $\dagger$ The surface tension of the pure solvents (choline chloride:urea: $66.3 \pm$ $0.2 \mathrm{mN} \mathrm{m}^{-1}$; choline chloride:glycerol: $63.6 \pm 0.4 \mathrm{mN} \mathrm{m}^{-1}$ ) and water $\left(72.1 \pm 0.3 \mathrm{mN} \mathrm{m}^{-1}\right)$ were measured as a control, and the values were found to be consistent with those previously published. ${ }^{11,12}$

All surfactants presented here show the typical behaviour of a surface-active compound, reducing the surface tension of the liquid with increasing concentration down to a minimum. Above the CMC, there is no significant change in the surface tension. This is indicative of micelles forming in the bulk phase

Table 2 CMC and limiting surface tension $\left(\gamma_{c}\right)$ of the dodecylsulfate surfactants in choline chloride:urea, choline chloride:glycerol and water

\begin{tabular}{|c|c|c|c|c|c|}
\hline \multirow[b]{2}{*}{ Surfactant } & \multicolumn{2}{|c|}{ Choline chloride:urea } & \multicolumn{2}{|c|}{ Choline chloride:glycerol } & \multirow{2}{*}{$\frac{\text { Water }}{\mathrm{CMC} / \mathrm{mM}}$} \\
\hline & $\mathrm{CMC} / \mathrm{mM}$ & $\gamma_{\mathrm{c}} / \mathrm{mN} \mathrm{m}^{-1}$ & $\mathrm{CMC} / \mathrm{mM}$ & $\gamma_{\mathrm{c}} / \mathrm{mN} \mathrm{m}^{-1}$ & \\
\hline LiDS & $2.4 \pm 0.2$ & $30.4 \pm 0.1$ & $4.2 \pm 0.3$ & $30.9=$ & $8.9^{38}$ \\
\hline SDS & $2 \pm 1^{12}$ & $27.8 \pm 1.3^{12}$ & $3.9 \pm 0.1$ & $31.3 \pm 0.3$ & $8.1^{39}$ \\
\hline EmimDS & $1.8 \pm 0.2$ & $30.4 \pm 0.1$ & $3.7 \pm 0.1$ & $31.5 \pm 0.2$ & - \\
\hline ChDS & $1.8 \pm 0.1$ & $30.7 \pm 0.2$ & $2.3 \pm 0.2$ & $31.3 \pm 0.3$ & $4.26^{40}$ \\
\hline $\mathrm{Mg}_{1 / 2} \mathrm{DS}$ & $1.2 \pm 0.1$ & $30.4 \pm 0.2$ & $2.1 \pm 0.2$ & $31.4 \pm 0.3$ & $1.76^{41}$ \\
\hline CsDS & $1.9 \pm 0.1$ & $32.1 \pm 0.3$ & $3.1 \pm 0.2$ & $31.1 \pm 0.1$ & $6.1^{38}$ \\
\hline BmimDS & $1.3 \pm 0.2$ & $31.2 \pm 0.2$ & $2.1 \pm 0.1$ & $31.5 \pm 0.2$ & $1.8^{42}$ \\
\hline
\end{tabular}

of the solvent, as the inflexion point in the surface tension corresponds with the CMC.

The CMC of dodecylsulfate surfactants with simple cations were found to be consistently lower than those in water for both DES, except for $\mathrm{Mg}_{1 / 2} \mathrm{DS}$ in choline chloride:glycerol. This was previously found for SDS in choline chloride:urea (CMC $=2 \pm$ $1 \mathrm{mM})^{12}$ and choline chloride:glycerol $(\mathrm{CMC}=3.9 \pm 0.1 \mathrm{mM})$ (see $\mathrm{ESI} \dagger$ ), where the CMC is considerably lower than the value in aqueous solution $(\mathrm{CMC}=8.1 \mathrm{mM}) \cdot{ }^{39}$ This suggests that the solubility of surfactant monomers in DES is considerably lower than in water, despite the lower polarity of DES. ${ }^{43}$ Previous investigations on the surface behaviour of cationic surfactants in choline chloride:glycerol showed that the CMC values of $\mathrm{C}_{n}$ TABs in this DES were higher than in water, which was assigned to the lower polarity of the solvent. ${ }^{11}$ Therefore, the mechanism of headgroup and counterion solvation has an effect on the solubility of the anionic monomers, leading to considerably lower CMCs than in water.

One of the most obvious changes in ionic surfactant behaviour as the counterion is varied is that the CMC of the surfactant in water changes as the counterion is changed. Counterions that have an increased binding to the surfactant headgroup in aqueous solution lower the CMC of the surfactant. ${ }^{22,44,45}$ Previous investigations of surfactant behaviour in ionic liquids assumed that the counterions would be completely exchanged due to the overwhelming number of solvent ions compared to native surfactant ions and thus counterion binding effects would be negligible. ${ }^{46}$ Such a hypothesis may be extended to DES due to the similarly strong ionic environment. However, our results demonstrate that this hypothesis is not valid for the systems presented here. As shown in Table 2, the surface behaviour of dodecylsulfate surfactants is affected by different counterions in choline chloride-based DES in a similar fashion to that in water. The CMC of monovalent inorganic counterions in choline chloride:urea and choline chloride:glycerol follows the trend: $\mathrm{CMC}_{\mathrm{LiDS}}<\mathrm{CMC}_{\mathrm{SDS}}<\mathrm{CMC}_{\mathrm{CSDS}}$, similar to that observed in aqueous solution. ${ }^{19,44,47,48}$ In the case of the divalent counterion, $\mathrm{Mg}^{2+}$, the CMCs of the solutions are considerably lower than in the case of monovalent counterions, as also found in water. ${ }^{41,44}$

In the case of organic counterions, the presence of a stronger lyophobe, $\mathrm{Bmim}^{+}$, remarkably decreases the CMC in comparison with the less lyophobic counterion, $\mathrm{Emim}^{+}$. This suggests that the solubility of the surfactant below the CMC depends on the lyophobicity of the organic counterpart, and may be related either to the strength of the counterion binding to the surfactant ion or the inability of that counterion to be solubilised in the highly H-bonded solvent. This can be corroborated with similar trends seen for amphiphilic ionic liquids in water; substituting the counterion for something more lyophobic reduces the CMC of the surfactant, but the free energy penalty for additional alkyl chain units in the counterion is much less than that seen for the surfactant tail. ${ }^{24,49}$ The CMC of ChDS in choline-based DES was found to be in-between the values presented for the inorganic and organic counterions, showing an intermediate ion binding in comparison. Despite the high concentration of choline in the solvent that could be expected to 
dominate the adsorption of ions at the micelle interface for all surfactants, our results clearly indicate that the native counterion of the surfactants still plays a role in the limiting surface tension of the system and CMC.

\section{Micellisation in the diluted regime}

The effect of counterions on the micellisation of these surfactants in DES was studied by comparing the aggregation of lithium, caesium, magnesium, 1-ethyl-3-methylimidazolium, 1-butyl-3methylimidazolium, and choline salts at different concentrations in choline chloride:urea and choline chloride:glycerol. Samples at low concentration, in the dilute regime, were measured and fitted to obtain information about the form factor of the micelles, avoiding contribution from intermicellar interactions. Previous investigations in analogous systems (sodium dodecylsulfate in $1: 2$ choline chloride : urea) showed negligible intermicellar interactions up to at least $\sim 45 \mathrm{mM} .^{19}$ Therefore we assume that the scattering from the concentrations presented here also contain no or negligible structure factor contribution. The results from the Guinier analysis of the SANS data of the different surfactants in the dilute regime are included in the ESI. $\dagger$ SANS data and best fits of these surfactants in choline chloride:urea and choline chloride:glycerol are presented in Fig. 2. The results from the fits are presented in Tables 3 and 4.

All surfactants studied here were found to form micelles at the measured concentrations. As previously reported for SDS in the choline chloride:urea eutectic mixture, anionic surfactants form elongated micelles in this solvent even at low concentrations. ${ }^{12}$ Unlike micelles in choline chloride:urea, where aspect ratios vary between 8.8 and 13.5 ( $R_{\mathrm{g}}$ varies between 88.5-101 $\AA$ ) in the dilute regime, micelles in choline chloride:glycerol were found to preferably form globular morphologies, with aspect ratios around $1.9\left(R_{\mathrm{g}}\right.$ varies between 17.1-17.9 $\left.\mathrm{\AA}\right)$.

The size and elongation of micelles in choline chloride:urea DES was found to depend on the surfactant counterion. While the minor axis of the micelles (equatorial axis) remains practically unchanged in all cases, variations in surfactant counterion produce changes in the aspect ratio of the micelles. Between the inorganic counterions, lithium and magnesium are shown to form the shorter micelles, whereas caesium shows longer micelles $(\sim 26 \%$ longer than LiDS micelles in the same solvent at the lowest concentration measured). The scattering of EmimDS, BmimDS and ChDS micelles also showed the presence of elongated micelles with variable elongation depending on the counterion. In this case, the smallest micelle aspect ratio at the lowest concentration is determined for EmimDS, while BminDS showed larger micelles ( 27\% longer). Micelles composed of ChDS seem to be comparable to those of EmimDS within error.

Surfactant concentration also affects the structure of the micelles, as previously shown for SDS in choline chloride:urea. ${ }^{19}$ In the dilute regime, increasing the concentration of surfactant leads to an increase in the aspect ratio of the micelle. The average increase in aspect ratio for all surfactants studied here is $\sim 24 \%$ with an increase in surfactant concentration from $25.1 \pm 3.0 \mathrm{mM}$ to $39.8 \pm 2.5 \mathrm{mM}$.

It is also observed that the volume fraction of micelles increases as the counterion is varied from $\mathrm{Li}^{+}$or $\mathrm{Mg}^{2+}$ to $\mathrm{Cs}^{+}$. Given that the size and shape of the micelles are determined from the shape of the scattering curve and not the intensity of the scattering, then for a given shape and size, the volume fraction reduces to the number density of micelles. This variation in the volume fraction of micelles is observed between $\mathrm{Emim}^{+}$

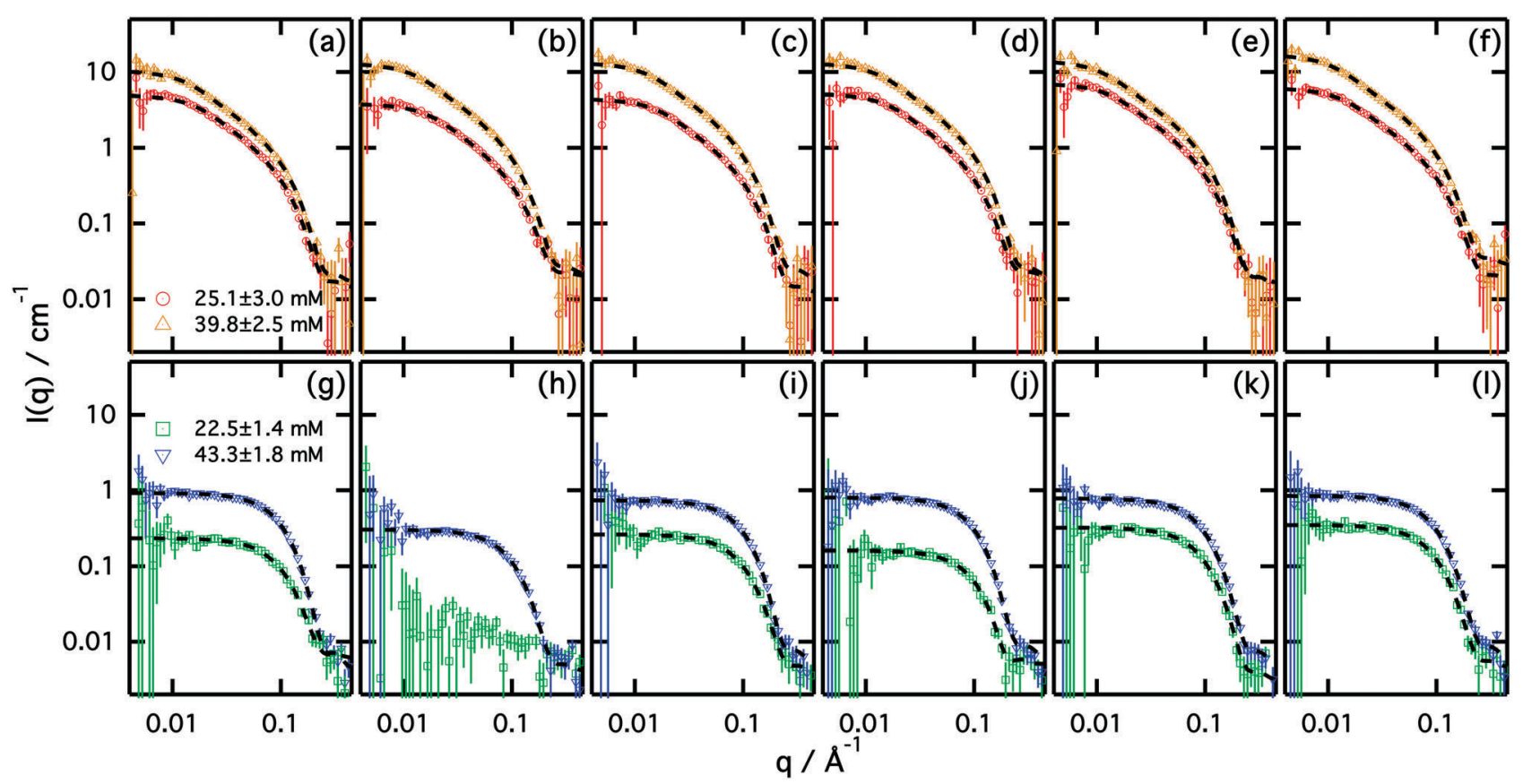

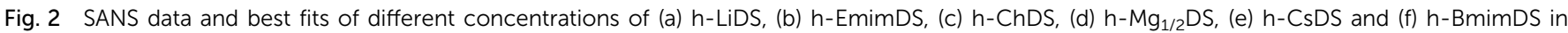

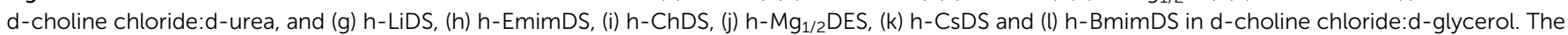
average concentration of surfactant in each solvent is presented in the plots a and g. Fits are represented as black-dashed lines. 
Table 3 Results from SANS fitting for two different concentrations (dilute regime) of different surfactants in choline chloride:urea. The fitted volume fraction corresponds to the value obtained from the $\mathrm{h}$-surfactant in $d$-solvent. The calculated volume fraction of micelles is presented for comparison with the results

\begin{tabular}{lrrrr}
\hline Surfactant & \multicolumn{1}{c}{$r_{\mathrm{eq}} / \AA$} & \multicolumn{1}{c}{$\mathrm{AR}$} & $\phi_{P(q)} / 10^{-2}$ & \multicolumn{1}{c}{$\phi_{\text {Calc }} / 10^{-2}$} \\
\hline $25.1 \pm 3.0 \mathrm{mM}$ & & & & \\
LiDS & $16.4 \pm 0.3$ & $8.9 \pm 0.5$ & $0.69 \pm 0.14$ & $0.62 \pm 0.04$ \\
EmimDS & $16.1 \pm 0.4$ & $8.8 \pm 0.5$ & $0.58 \pm 0.08$ & $0.60 \pm 0.09$ \\
ChDS & $15.8 \pm 0.4$ & $9.4 \pm 0.3$ & $0.68 \pm 0.08$ & $0.69 \pm 0.03$ \\
Mg $_{1 / 2}$ DS & $16.6 \pm 0.3$ & $9.2 \pm 0.2$ & $0.68 \pm 0.07$ & $0.71 \pm 0.10$ \\
CsDS & $16.3 \pm 0.4$ & $11.2 \pm 0.2$ & $0.74 \pm 0.08$ & $0.82 \pm 0.10$ \\
BmimDS & $15.9 \pm 0.4$ & $11.2 \pm 0.3$ & $0.78 \pm 0.08$ & $0.75 \pm 0.07$
\end{tabular}

$39.8 \pm 2.5 \mathrm{mM}$

$\begin{array}{lllll}39.8 \pm 2.5 & \mathrm{mM} & & \\ \text { LiDS } & 16.2 \pm 0.2 & 11.5 \pm 0.4 & 1.1 \pm 0.2 & 1.2 \pm 0.2 \\ \text { EmimDS } & 16.6 \pm 0.3 & 11.7 \pm 0.4 & 1.3 \pm 0.1 & 1.3 \pm 0.2 \\ \text { ChDS } & 16.5 \pm 0.2 & 11.3 \pm 0.2 & 1.4 \pm 0.1 & 1.4 \pm 0.1 \\ \mathrm{Mg}_{1 / 2} \text { DS } & 16.3 \pm 0.2 & 12.0 \pm 0.2 & 1.4 \pm 0.2 & 1.3 \pm 0.2 \\ \text { CsDS } & 16.6 \pm 0.4 & 13.5 \pm 0.4 & 1.3 \pm 0.1 & 1.4 \pm 0.1 \\ \text { BmimDS } & 16.5 \pm 0.2 & 13.7 \pm 0.3 & 1.5 \pm 0.1 & 1.6 \pm 0.2\end{array}$

Table 4 Results from SANS fitting for two different concentrations (dilute regime) of different surfactants in choline chloride:glycerol. The fitted volume fraction corresponds to the value obtained from the $h$-surfactant in $d$-solvent. The calculated volume fraction of micelles is presented for comparison with the results

\begin{tabular}{lllll}
\hline Surfactant & $r_{\mathrm{eq}} / \AA$ & $\mathrm{AR}$ & $\phi_{P(q)} / 10^{-2}$ & $\phi_{\mathrm{Calc}} / 10^{-2}$ \\
\hline $22.5 \pm 1.4 \mathrm{mM}$ & & & \\
LiDS & $16.4 \pm 0.7$ & $1.87 \pm 0.11$ & $0.15 \pm 0.06$ & $0.26 \pm 0.05$ \\
EmimDS & - & - & - & $0.14 \pm 0.04$ \\
ChDS & $16.6 \pm 0.8$ & $1.92 \pm 0.12$ & $0.18 \pm 0.04$ & $0.17 \pm 0.01$ \\
Mg $_{1 / 2}$ DS & $19.6 \pm 1.8$ & $1.30 \pm 0.31$ & $0.15 \pm 0.04$ & $0.24 \pm 0.03$ \\
CSDS & $17.1 \pm 0.4$ & $1.89 \pm 0.07$ & $0.18 \pm 0.06$ & $0.29 \pm 0.08$ \\
BmimDS & $16.9 \pm 0.3$ & $1.93 \pm 0.07$ & $0.22 \pm 0.04$ & $0.28 \pm 0.02$
\end{tabular}

$43.3 \pm 1.8 \mathrm{mM}$

$\begin{array}{lllll}\text { LiDS } & 16.8 \pm 0.6 & 1.80 \pm 0.07 & 0.64 \pm 0.04 & 0.80 \pm 0.02\end{array}$

$\begin{array}{lllll}\text { EmimDS } & 17.1 \pm 0.4 & 1.78 \pm 0.08 & 0.38 \pm 0.03 & 0.61 \pm 0.12\end{array}$

$\begin{array}{lllll}\text { ChDS } & 17.3 \pm 0.2 & 1.70 \pm 0.04 & 0.42 \pm 0.05 & 0.67 \pm 0.06\end{array}$

$\begin{array}{lllll}\mathrm{Mg}_{1 / 2} \mathrm{DS} & 17.3 \pm 0.2 & 1.71 \pm 0.08 & 0.45 \pm 0.03 & 0.68 \pm 0.05\end{array}$

$\begin{array}{lllll}\mathrm{CsDS} & 17.2 \pm 0.3 & 1.87 \pm 0.05 & 0.43 \pm 0.03 & 0.90 \pm 0.13\end{array}$

$\begin{array}{lllll}\text { BmimDS } & 16.9 \pm 0.2 & 1.90 \pm 0.03 & 0.43 \pm 0.04 & 0.63 \pm 0.07\end{array}$

and $\mathrm{Bmim}^{+}$counterions and can be correlated with the CMC of those surfactants in the solvent.

In the case of the choline chloride:glycerol eutectic mixture, we see that the surfactants studied here form globular, rather than elongated, micelles. Although there seems to be an underlying trend in the results, the uncertainties in the fitted parameters mean that solid conclusions cannot be drawn, except that that the counterion has a weaker effect on the micellization in this solvent.

\section{Micellisation in the concentrated regime}

A high concentration of the various surfactants in choline chloride:urea was measured to study the behaviour of the amphiphiles in the concentrated regime. As the concentration of surfactant increases, intermicellar interactions begin to appear and affect the scattering. Although previous investigations have shown that the structure factor contribution is relatively low at these concentrations $\left(\phi_{S(q)}=2.8 \times 10^{-2}\right.$ at $81.3 \mathrm{mM}$ of SDS in choline chloride:urea), accounting for such a contribution is necessary in order to obtain reliable structural information about the micelles. In order to fit these data, we have used a model that combines the uniform ellipsoid form factor and the modified hard-sphere approximation, described previously. ${ }^{19,28}$ SANS data and best fits are presented in Fig. 3. The results from the fits are included in Table 5. Furthermore, these fits are compared with the contribution from the form factor to the model (i.e. $S(q)=1$ ).

Our results clearly show that the form factor model is not sufficient to model the scattering of the more concentrated systems, and the disagreement between the data and the fits is considerable. Thus a structure factor must be included in order to account for the intermicellar contribution. Although the implemented model cannot be used as an analytic approach to investigate those interactions, the intermicellar scattering can be accounted for, and therefore the form factor of the aggregates can be determined.

For all of the surfactants presented here, elongated micelles are found in choline chloride:urea as the concentration of surfactant is increased in the dilute regime. As shown in the results, the equatorial radius of the micelles is comparable between different counterions at this concentration. The aspect ratio, however, varies with surfactant counterion in a similar way as presented above for the low concentrations, showing the largest values for CsDS and BmimDS (only values for solutions with a similar volume fraction of micelles were compared here).

As concentration increases in choline chloride:urea, the micelles were generally seen to elongate in the dilute regime. When the concentration is further increased, a critical point is reached whereupon the micelles begin to shrink. Close to the reversal point, which occurs between the intermediate concentration and the high concentration studied here, the aspect ratio for CsDS, for instance, increases from $11.2 \pm 0.2$ (at $25.1 \pm 3.0 \mathrm{mM}$ ) to $13.5 \pm 0.4$ (at $39.8 \pm 3.5 \mathrm{mM}$ ) and then retracts to $10.6 \pm 0.2$ (at $196 \pm 11 \mathrm{mM}$ ). This feature was previously observed and reported for SDS in choline chloride:urea. ${ }^{19}$

The results presented in Fig. 3 show a considerable difference between the model that uses only the form factor and the model that implements the structure factor and form factor. The difference in the scattering cross section observed at low $q$ is commonly attributed to repulsive interactions between aggregates. ${ }^{34}$ Furthermore, the values of the volume fraction of scatterers and that one provided by the structure factor are decoupled. The values of the structure factor contribution are consistently higher, showing that a pure hard sphere model, which should have the form and structure factor volume fractions equal, is insufficient to account for those interactions. These higher values of the structure factor volume fraction are attributed to repulsive interactions that may arise from electrostatic forces between micelles.

\section{Discussion}

\section{Counterion condensation}

The aggregation of dodecylsulfate surfactants in choline chloride: urea shows the formation of elongated micelles throughout the 


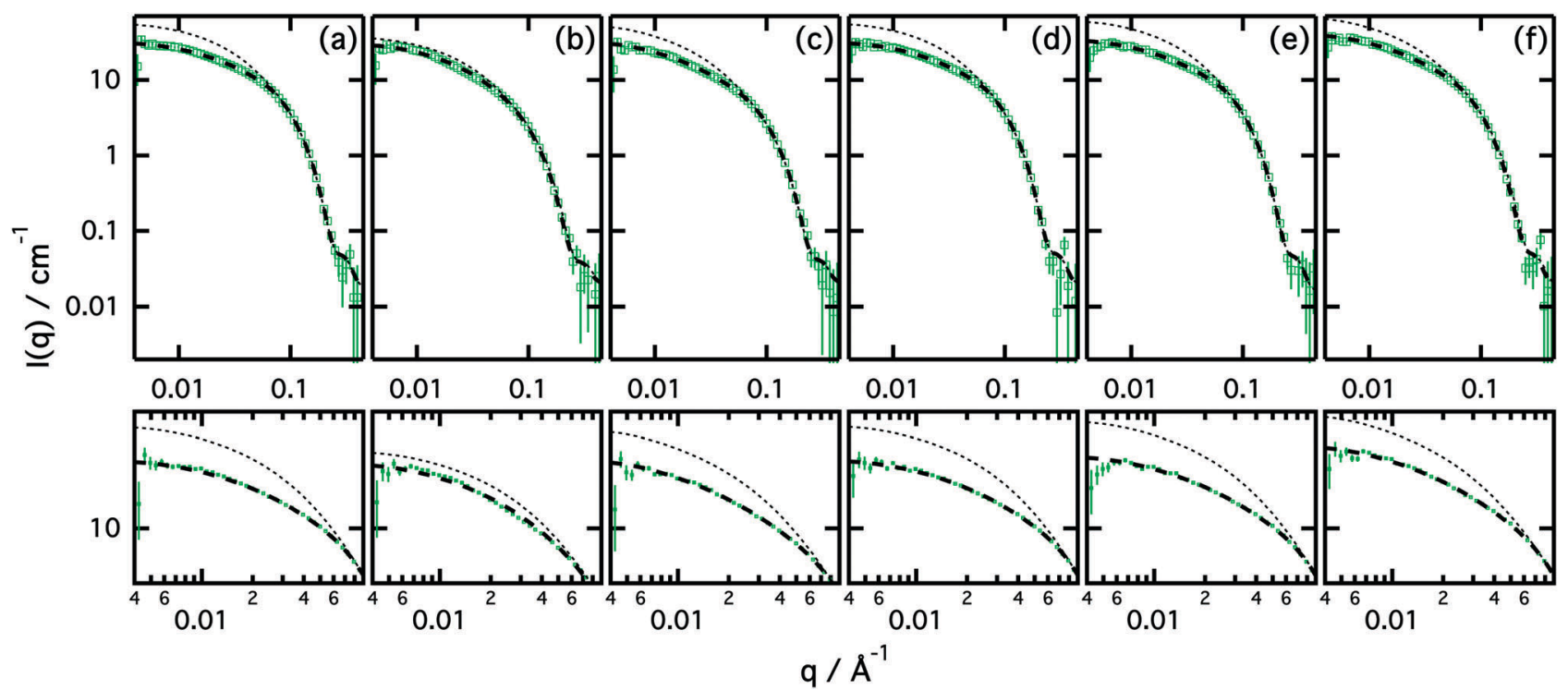

Fig. 3 SANS data and best fits for an averaged concentration of $196 \pm 11 \mathrm{mM}$ of (a) h-LiDS, (b) h-EmimDS, (c) h-ChDS, (d) h-Mg $1 / 2$ DS, (e) h-CsDS and (f) $\mathrm{h}$-BmimDS in d-choline chloride:d-urea. Fits using the model that combines form factor and structure factor are represented as black-dashed lines. Black-dotted lines show the form factor contribution to the model. The plots below zoom into the low- $q$ region of the data and fits.

Table 5 Parameters from fitting SANS data in Fig. 3, for solutions of all surfactants in choline chloride:urea at an averaged concentration of $196 \pm 11 \mathrm{mM}$. The fitted volume fraction corresponds to the value obtained from the h-surfactant in $d$-solvent. The calculated volume fraction of micelles, obtained from the volume of surfactant in the micellar phase, is presented for comparison with the results

\begin{tabular}{lrrrrrr}
\hline Surfactant $r_{\mathrm{eq}} / \AA$ & \multicolumn{1}{c}{$\mathrm{AR}$} & \multicolumn{1}{c}{$\phi_{P(q)} / 10^{-2}$} & $\phi_{\text {Calc }} / 10^{-2}$ & $R_{\mathrm{eff}} / \AA$ & $\phi_{S(q)} / 10^{-2}$ \\
\hline LiDS & $16.7 \pm 0.5$ & $9.6 \pm 0.7$ & $5.5 \pm 0.2$ & $5.6 \pm 0.4$ & 37.2 & $7.4 \pm 0.3$ \\
EmimDS & $17.6 \pm 0.2$ & $10.3 \pm 0.4$ & $3.6 \pm 0.1$ & $3.5 \pm 0.2$ & 38.3 & $3.7 \pm 0.1$ \\
$\mathrm{ChDS}$ & $17.5 \pm 0.4$ & $12.4 \pm 0.8$ & $4.1 \pm 0.1$ & $4.9 \pm 0.3$ & 40.5 & $6.4 \pm 0.1$ \\
$\mathrm{Mg}_{1 / 2} \mathrm{DS}$ & $17.8 \pm 0.3$ & $9.3 \pm 0.2$ & $5.6 \pm 0.2$ & $5.8 \pm 0.3$ & 37.5 & $7.3 \pm 0.2$ \\
CsDS & $17.8 \pm 0.4$ & $10.6 \pm 0.2$ & $5.4 \pm 0.2$ & $5.9 \pm 0.5$ & 39.3 & $7.5 \pm 0.6$ \\
BmimDS & $18.0 \pm 0.4$ & $10.6 \pm 0.3$ & $5.4 \pm 0.2$ & $6.4 \pm 0.7$ & 39.6 & $6.9 \pm 0.2$
\end{tabular}

range of concentrations investigated. Elongated micelles contain surfactant molecules packed with smaller headgroup areas than found in spherical or globular micelles. ${ }^{7}$ It was initially speculated that this was due to surfactant-urea interactions at the micellar interface, ${ }^{50}$ but assuming that the surface is not enriched in urea relative to the bulk, ${ }^{51}$ counterion binding to the micelle surface must be the most significant factor in this solvent; a primary solvation shell with condensed counterions is sufficient to rationalise the observed elongation behaviour. Micelle morphology, apparent fractional charge, and intermicellar interactions strictly depend on the nature of the ionic surfactant counterion and electrolytes in solution. The condensation of counterions onto an ionic micelle interface reduces the interfacial charge density and thus affects the packing parameter of individual monomers. ${ }^{7,36,48,52-55}$ The nature of the Stern layer, which surrounds the micelle, is characterised by the presence of oppositely charged counterions that affect the charge density of the micelle. The counterions located in this layer are electrostatically bound to the micelle, affecting the electrostatic repulsion between headgroups. This binding in aqueous solution is governed by the hydrated ionic size of the counterion and headgroup, which are in turn described by the Hofmeister series. ${ }^{48,56}$ In particular, for cations in the presence of an alkylsulfate group in aqueous solution the series is (from strongly bound cations to strongly hydrated cations): $\mathrm{Cs}^{+} \approx \mathrm{Ch}^{+}>\mathrm{Na}^{+}>\mathrm{Li}^{+}{ }^{+56-58}$ Furthermore, the co-surfactancy of organic counterions with lyophobic moieties has been investigated, showing that not only does the charge screening produced by the condensation of the salts affect the micellization, but there is also a contribution from the counterion acting as a co-surfactant which changes the monomer packing by penetrating into the micelle core. ${ }^{52,59-62}$

Varying the native counterion of the surfactant changes the micellisation of the surfactant, forming shorter aggregates for lithium than for caesium. This fact is suggestive of caesium counterions showing a more significant condensation at the micelle surface (tightly bound), therefore more strongly modifying the headgroup effective area and allowing the formation of more elongated aggregates than in the case of lithium (strongly solvated). Variations in the micelle morphology thus reflect the trends in the CMCs, and suggests that choline chloride:urea is not a good solvent for small inorganic cations, leading to greater counterion binding on the micelles than observed in water. In the case of surfactants in aqueous solution the solvated size of the counterion plays a role in micellisation, contributing to both the structure of the micelles and the interaction between them. We propose that this same mechanism is at work in the case of micellisation in choline chloride:urea DES, where the variation in micelle elongation correlates with the ion-pair formation, as previously reported for alkylsulfate amphiphiles. ${ }^{56,63}$ Similar ion binding strengths are seen in aqueous solutions of analogous systems, ${ }^{22,48,56}$ although elongated micelles are generally only found for dodecylsulfate anions in the presence 
of added salts, ${ }^{53}$ suggesting the extent of counterion binding to the micelles is greater in choline chloride:urea than in water.

Similarly, the ability of the solvent to solvate the various organic counterions controls the adsorption of these species onto the micelle interface. This factor is intrinsically dependent upon the presence of lyophobic moieties in the counterion. BmimDS in choline chloride:urea has been shown to form more elongated micelles than EmimDS micelles, suggesting that the $\mathrm{Bmim}^{+}$counterion has more affinity to stay in the Stern layer than $\mathrm{Emim}^{+}$and therefore provide a more efficient charge screening. This behaviour has been previously reported for the aggregation of SDS in aqueous solutions containing ionic liquids, where longer lyophobic moieties on the imidazolium cation promote the growth of the micelles by acting as co-surfactants which insert into the micelle and alter the average headgroup area and tail arrangement. ${ }^{59,64,65}$ Similarly, the lyophobicity of the alkylmethylimidazolium cation has been also correlated to the interaction of those with proteins in hydrated ionic liquids. ${ }^{66}$ Thus in the case of organic counterions, not only the charge neutralisation plays a role in the micellisation in choline chloride:urea DES, but also the lyophobicity of such counterions. ${ }^{64}$ Furthermore, the validity of the Hofmeister series have been explored in ionic liquids, and it is suggested that Hofmeister series may be a good frame to explore ion-affinity in these solvents. ${ }^{67,68}$ However those investigations also remark the high complexity of the ionic environment in these novel solvents, and further study needs to be done to provide a unifying Hofmeister theory that satisfies water and ionic solvents. ${ }^{66,67}$ Similarly, the behaviour in DES appears to be complex and, although the same underlying mechanism seems to be at work here, further investigations will be needed to validate the applicability of the Hofmeister series to the behaviour of macromolecules in DES.

The micellisation of ChDS is particularly interesting because it demonstrates that choline counterions also bind to the micelle headgroup. Again, the micellisation in choline chloride:urea is characterised by the low interfacial charge density and the formation of elongated micelles. This suggests that in the presence of a certain concentration of dissociated choline in any system, choline is adsorbed to the micelle interface, whether it is either the native surfactant counterion or from the solvent. In this scenario, the micellisation of other dodecylsulfate surfactants (e.g. LiDS or CsDS) will be affected by a competition between dissociated choline cations from the solvent and the native counterion of the surfactant. In fact, previous investigations hypothesised that choline may be partially adsorbed to the surfactant headgroups and participate, together with sodium counterions, in the micellisation of SDS in choline chloride:urea. ${ }^{19}$

\section{Intermicellar interactions}

As found in aqueous solution, increasing surfactant concentration leads to micelle-micelle correlations that begin to affect the scattering. ${ }^{18}$ A high surfactant concentration in choline chloride: urea has been found to require the application of a model that accounts for a structure factor contribution. The formation of elongated micelles at such concentrations is still observed, although the results show a reversion in the micelle growth above a critical concentration, with the formation of shorter micelles. This decrease is correlated with an increase in the structure factor volume fraction. The reversion in the micelle growth with concentration was previously hypothesised to arise from the limited amount of choline available to adsorb at the interface and the depletion of counterion in the Stern layer. ${ }^{19}$ Although at this stage we show that such behaviour is also counterion-dependent (showing more elongated micelles in the case of CsDS than for LiDS or $\mathrm{Mg}_{1 / 2} \mathrm{DS}$ at comparable micelle volume fractions), the underlying mechanism is still not well understood.

\section{Counterion solvation and micelle morphology}

Dodecylsulfate surfactants are commonly known to form strongly repulsive globular micelles in pure water due to the counterion depletion at the interface and the resulting high chargedensity. ${ }^{36,69}$ We show that choline chloride-based DES micelles appear to be more elongated, particularly in choline chloride:urea, and the intermicellar interactions are largely diminished. These results can be again interpreted as a different interaction between the headgroup of the surfactant and the cations present in the system. Whereas water would provide a suitable environment for counterion dissociation, choline chloride:urea may show a lower solubility of cations and therefore those will favourably be allocated at the micelle interface. Choline chloride:glycerol would be an environment with intermediate counterion affinity between water and the urea-based DES, potentially driven by the solubility of the cation in glycerol. ${ }^{70}$ This would be consistent with recent findings from Faraone et al., who demonstrated that the dynamic nanostructure of the choline chloride:glycerol DES is strongly glycerol-dominated, with choline merely occupying voids in the glycerol network rather than being defined by choline-hydrogen bond donor, and choline-halide interactions. ${ }^{71}$ Considering this ability to solubilise more counterions, the glycerolbased DES would be more prone to promote the depletion of counterions from the micelle interface than the urea-based solvent. Therefore, micelles in this scenario appear to present a higher charge density at the Stern layer and adopt globular morphologies instead of elongated.

\section{Conclusions}

We have shown that the behaviour of a series of anionic dodecylsulfate surfactants, with inorganic (lithium, caesium and magnesium) and organic (butylmethylimidazolium, ethylmethylimidazolium and choline) counterions, varies considerably between choline chloride:urea, choline chloride:glycerol and water. The general trend of the amphiphiles investigated here shows that the CMCs in both DES are below those in water. Since the polarity of the eutectic mixtures is lower than that of water, ${ }^{43}$ the surfactant CMCs would be expected to be higher in DES. However, our results show otherwise and therefore the surfactant monomers are less soluble in DES than in water. This indicates that cations are more tightly bound to 
the headgroup in these DES than in water, where the strongest ion-ion interaction appears in the urea-based system.

These results also correlate with the observations in micellar structure and intermicellar interactions. The aggregation of those surfactants in the bulk phase is presented for both eutectic solvents. Micelle morphology is affected by the surfactant counterion and solvent. Whereas dodecylsulfate micelles in choline chloride glycerol are globular, urea-based DES supports the formation of weakly interacting, elongated micelles. Variations on aggregate morphology are also found with varying the native counterion of surfactant, where those results have been rationalised through the interaction of the headgroup with charged counterparts at the solvation shell.

The results presented here demonstrate the existence of specific charge-based interactions in DES. As in aqueous solution, weakly-solvated ions are more prone to interaction with weakly-solvated counterparts, and vice versa, and DES interestingly show a similar behaviour in the present case of the surfactant aggregation. Overall, the conclusions of this study are applicable to a general theory on the behaviour of surfactants and proteins in DES, where ion condensation effects constitute a valid framework for understanding the behaviour of macromolecules in these exotic environments.

\section{Conflicts of interest}

There are no conflicts of interest to declare.

\section{Acknowledgements}

The authors would like to thank the European Spallation Source and the University of Bath Alumni Fund for funding A. S.-F. We thank the UK ISIS Pulsed Neutron and Muon source at the Rutherford Appleton Laboratory and the UK Engineering and Physical Sciences Research Council (EPSRC) for supporting O. S. H. in the Centre for Doctoral Training in Sustainable Chemical Technologies at the University of Bath (EP/L016354/1; STFC Studentship Agreement 3578). We also thank ISIS Pulsed Neutron and Muon source for the awarded beamtime (experiment number RB1620126). The authors would also like to thank Diamond Light Source (UK) for the access to the laboratories and equipment for performing surface tension measurements. This work benefited from the use of the SasView application, originally developed under NSF Award DMR-0520547. SasView also contains code developed with funding from the EU Horizon 2020 programme under the SINE2020 project Grant No. 654000.

\section{References}

1 E. L. Smith, A. P. Abbott and K. S. Ryder, Chem. Rev., 2014, 114, 11060-11082.

2 O. S. Hammond, D. T. Bowron, A. J. Jackson, T. Arnold, A. Sanchez-Fernandez, N. Tsapatsaris, V. Garcia Sakai and K. J. Edler, J. Phys. Chem. B, 2017, 121, 7473-7483.

3 O. S. Hammond, D. T. Bowron and K. J. Edler, Green Chem., 2016, 18, 2736-2744.
4 C. F. Araujo, J. A. P. Coutinho, M. M. Nolasco, S. F. Parker, P. J. A. Ribeiro-Claro, S. Rudic, B. I. G. Soares and P. D. Vaz, Phys. Chem. Chem. Phys., 2017, 19, 17998-18009.

5 S. Zahn, B. Kirchner and D. Mollenhauer, ChemPhysChem, 2016, 17, 3354-3358.

6 A. Paiva, R. Craveiro, I. Aroso, M. Martins, R. L. Reis and A. R. C. Duarte, ACS Sustainable Chem. Eng., 2014, 2, 1063-1071.

7 J. N. Israelachvili, D. J. Mitchell and B. W. Ninham, J. Chem. Soc., Faraday Trans. 2, 1976, 72, 1525.

8 R. Hargreaves, D. T. Bowron and K. Edler, J. Am. Chem. Soc., 2011, 133, 16524-16536.

9 J. A. Long, B. M. Rankin and D. Ben-Amotz, J. Am. Chem. Soc., 2015, 137, 10809-10815.

10 R. Nagarajan and E. Ruckenstein, Langmuir, 1991, 7, 2934-2969.

11 A. Sanchez-Fernandez, T. Arnold, A. J. Jackson, S. L. Fussell, R. K. Heenan, R. A. Campbell and K. J. Edler, Phys. Chem. Chem. Phys., 2016, 18, 33240-33249.

12 T. Arnold, A. J. Jackson, A. Sanchez-Fernandez, D. Magnone, A. E. Terry and K. J. Edler, Langmuir, 2015, 31, 12894-12902.

13 Z. Chen, S. McDonald, P. FitzGerald, G. G. Warr and R. Atkin, J. Colloid Interface Sci., 2017, 506, 486-492.

14 L. Sapir, C. B. Stanley and D. Harries, J. Phys. Chem. A, 2016, 120, 3253-3259.

15 A. Sanchez-Fernandez, K. J. Edler, T. Arnold, D. Alba Venero and A. J. Jackson, Phys. Chem. Chem. Phys., 2017, 19, 8667-8670.

16 S. J. Bryant, R. Atkin and G. G. Warr, Langmuir, 2017, 33, 6878-6884.

17 S. J. Bryant, R. Atkin and G. G. Warr, Soft Matter, 2016, 12, 1645-1648.

18 J. B. Hayter and J. Penfold, J. Chem. Soc., Faraday Trans. 1, 1981, 77, 1851.

19 A. Sanchez-Fernandez, K. J. Edler, T. Arnold, R. K. Heenan, L. Porcar, N. J. Terrill, A. E. Terry and A. J. Jackson, Phys. Chem. Chem. Phys., 2016, 18, 14063-14073.

20 A. P. Abbott, G. Capper, D. L. Davies, R. K. Rasheed and V. Tambyrajah, Chem. Commun., 2003, 70-71, DOI: 10.1039/ B210714G.

21 A. P. Abbott, R. C. Harris, K. S. Ryder, C. D’Agostino, L. F. Gladden and M. D. Mantle, Green Chem., 2011, 13, 82-90.

22 J. R. Lu, A. Marrocco, T. J. Su, R. K. Thomas and J. Penfold, J. Colloid Interface Sci., 1993, 158, 303-316.

23 K. Shinoda and T. Hirai, J. Phys. Chem., 1977, 81, 1842-1845. 24 P. Brown, C. P. Butts, J. Eastoe, D. Fermin, I. Grillo, H.-C. Lee, D. Parker, D. Plana and R. M. Richardson, Langmuir, 2012, 28, 2502-2509.

25 R. K. Heenan, S. E. Rogers, D. Turner, A. E. Terry, J. Treadgold and S. M. King, Neutron News, 2011, 22, 3.

26 O. Arnold, J. C. Bilheux, J. M. Borreguero, A. Buts, S. I. Campbell, L. Chapon, M. Doucet, N. Draper, R. Ferraz Leal, M. A. Gigg, V. E. Lynch, A. Markvardsen, D. J. Mikkelson, R. L. Mikkelson, R. Miller, K. Palmen, P. Parker, G. Passos, T. G. Perring, P. F. Peterson, S. Ren, M. A. Reuter, A. T. Savici, J. W. Taylor, R. J. Taylor, R. Tolchenov, W. Zhou and J. Zikovsky, Nucl. Instrum. Methods Phys. Res., Sect. A, 2014, 764, 156-166.

27 G. D. Wignall and F. S. Bates, J. Appl. Crystallogr., 1987, 20, 28-40. 
28 A. Sanchez-Fernandez, O. S. Hammond, A. J. Jackson, T. Arnold, J. Doutch and K. J. Edler, Langmuir, 2017, 33, 14304-14314.

29 J. S. Pedersen, Adv. Colloid Interface Sci., 1997, 70, 171-210. 30 S. S. Berr, E. Caponetti, J. S. Johnson, R. R. M. Jones and L. J. Magid, J. Phys. Chem., 1986, 90, 5766-5770.

31 M. Doucet, J. H. Cho, G. Alina, J. Bakker, W. Bouwman, P. Butler, K. Campbell, M. Gonzales, R. Heenan, A. Jackson, P. Juhas, S. King, P. Kienzle, J. Krzywon, A. Markvardsen, T. Nielsen, L. O'Driscoll, W. Potrzebowski, R. Ferraz Leal, T. Richter, P. Rozycko and A. Washington, SasView version 4.1, 2017, DOI: 10.5281/zenodo.438138.

32 Y. Chevalier and T. Zemb, Rep. Prog. Phys., 1990, 53, 279.

33 J.-P. Hansen and J. B. Hayter, Mol. Phys., 1982, 46, 651-656.

34 J. B. Hayter and J. Penfold, Mol. Phys., 1981, 42, 109-118.

35 J. K. Percus and G. J. Yevick, Phys. Rev., 1958, 110, 1-13.

36 Y. C. Liu, S. H. Chen and R. Itri, J. Phys.: Condens. Matter, 1996, 8, A169-A187.

37 V. K. Aswal and P. S. Goyal, Chem. Phys. Lett., 2003, 368, 59-65.

38 P. Mukerjee, K. Mysels and P. Kapauan, J. Phys. Chem., 1967, 71, 4166-4175.

39 P. H. Elworthy and K. J. Mysels, J. Colloid Interface Sci., 1966, 21, 331-347.

40 R. Klein, M. Kellermeier, D. Touraud, E. Müller and W. Kunz, J. Colloid Interface Sci., 2013, 392, 274-280.

41 M. Hato, M. Tahara and Y. Suda, J. Colloid Interface Sci., 1979, 72, 458-464.

42 J. Jiao, B. Dong, H. Zhang, Y. Zhao, X. Wang, R. Wang and L. Yu, J. Phys. Chem. B, 2012, 116, 958-965.

43 A. Pandey, R. Rai, M. Pal and S. Pandey, Phys. Chem. Chem. Phys., 2014, 16, 1559-1568.

44 S. Pandey, R. P. Bagwe and D. O. Shah, J. Colloid Interface Sci., 2003, 267, 160-166.

45 S. S. Berr, M. J. Coleman, R. R. M. Jones and J. S. Johnson, J. Phys. Chem., 1986, 90, 6492-6499.

46 D. F. Evans, A. Yamauchi, R. Roman and E. Z. Casassa, J. Colloid Interface Sci., 1982, 88, 89-96.

47 Y. Marcus, Chem. Rev., 1988, 88, 1475-1498.

48 J. V. Joshi, V. K. Aswal and P. S. Goyal, J. Phys.: Condens. Matter, 2007, 19, 196219.

49 P. Brown, C. Butts, R. Dyer, J. Eastoe, I. Grillo, F. Guittard, S. Rogers and R. Heenan, Langmuir, 2011, 27, 4563-4571.
50 W. Bruning and A. Holtzer, J. Am. Chem. Soc., 1961, 83, 4865-4866.

51 L. S. Romsted, J. Zhang, I. M. Cuccovia, M. J. Politi and H. Chaimovich, Langmuir, 2003, 19, 9179-9190.

52 C. A. Dreiss, Soft Matter, 2007, 3, 956-970.

53 M. Bergstrom and J. S. Pedersen, Phys. Chem. Chem. Phys., 1999, 1, 4437-4446.

54 L. Arleth, M. Bergström and J. S. Pedersen, Langmuir, 2002, 18, 5343-5353.

55 K. Kuperkar, L. Abezgauz, K. Prasad and P. Bahadur, J. Surfactants Deterg., 2010, 13, 293-303.

56 N. Vlachy, B. Jagoda-Cwiklik, R. Vácha, D. Touraud, P. Jungwirth and W. Kunz, Adv. Colloid Interface Sci., 2009, 146, 42-47.

57 A. A. Tietze, F. Bordusa, R. Giernoth, D. Imhof, T. Lenzer, A. Maaß, C. Mrestani-Klaus, I. Neundorf, K. Oum, D. Reith and A. Stark, ChemPhysChem, 2013, 14, 4044-4064.

58 H. I. Okur, J. Hladílková, K. B. Rembert, Y. Cho, J. Heyda, J. Dzubiella, P. S. Cremer and P. Jungwirth, J. Phys. Chem. B, 2017, 121, 1997-2014.

59 P. A. Hassan, S. R. Raghavan and E. W. Kaler, Langmuir, 2002, 18, 2543-2548.

60 P. A. Hassan, G. Fritz and E. W. Kaler, J. Colloid Interface Sci., 2003, 257, 154-162.

61 T. H. Ito, R. K. Rodrigues, W. Loh and E. Sabadini, Langmuir, 2015, 31, 6020-6026.

62 Z. Lin, J. J. Cai, L. E. Scriven and H. T. Davis, J. Phys. Chem., 1994, 98, 5984-5993.

63 V. E. Haverd and G. G. Warr, Langmuir, 2000, 16, 157-160.

64 S. Javadian, F. Nasiri, A. Heydari, A. Yousefi and A. A. Shahir, J. Phys. Chem. B, 2014, 118, 4140-4150.

65 J.-H. Lin and S.-S. Hou, J. Colloid Interface Sci., 2016, 474, 78-87.

66 H. Zhao, S. M. Campbell, L. Jackson, Z. Song and O. Olubajo, Tetrahedron: Asymmetry, 2006, 17, 377-383.

67 Z. Yang, J. Biotechnol., 2009, 144, 12-22.

68 A. Kumar and P. Venkatesu, Int. J. Biol. Macromol., 2014, 63, 244-253.

69 J. B. Hayter and J. Penfold, Colloid Polym. Sci., 1983, 261, 1022-1030.

70 J. Burgess, Metal ions in solution, Ellis Horwood, 1978.

71 A. Faraone, D. V. Wagle, G. A. Baker, E. C. Novak, M. Ohl, D. Reuter, P. Lunkenheimer, A. Loidl and E. Mamontov, J. Phys. Chem. B, 2018, 122, 1261-1267. 